\title{
Formation of polycyclic aromatic hydrocarbon grains using anthracene and their stability under UV irradiation
}

\author{
Midori Saito ${ }^{1}$, Itsuki Sakon ${ }^{2}$, Chihiro Kaito $^{3}$, and Yuki Kimura ${ }^{4}$ \\ ${ }^{1}$ Institute for Molecular Science, National Institutes of Natural Sciences, Okazaki, Aichi 444-8585, Japan \\ ${ }^{2}$ Department of Astronomy, School of Science, University of Tokyo, 7-3-1 Hongo, Bunkyo-ku, Tokyo 113-0033, Japan \\ ${ }^{3}$ Laboratory for Nano-Structure Science, Department of Physics, Ritsumeikan University, \\ 1-1-1 Nojihigashi, Kusatsu-shi, Shiga 525-8577, Japan \\ ${ }^{4}$ Department of Earth and Planetary Materials Science, Graduate School of Science, Tohoku University, Sendai 980-8578, Japan
}

(Received July 28, 2008; Revised November 21, 2008; Accepted December 5, 2008; Online published February 12, 2010)

\begin{abstract}
It has been known for some time that the properties of a material are changed in nanometer size. Since it is nontrivial that polycyclic aromatic hydrocarbon $(\mathrm{PAH})$ grains at the nanometer scale show similar properties with that of macroscopic scale, PAH grains were synthesized by a gas evaporation method and were analyzed using a transmission electron microscope and their UV-Vis and mid-IR spectra were measured. Results from these experiments showed that condensed anthracene grains from the gas phase had a planar form and showed similar infrared peaks compared to anthracene molecules, but with somewhat wider bands and different relative intensities. Some new features were also observed. The anthracene molecules formed photodimer in their grain and showed different infrared features after UV irradiation. This would be the first report concerning nanometersized PAH grains.
\end{abstract}

Key words: PAH, infrared, dust, laboratory experiment.

\section{Introduction}

After the discovery of unidentified infrared (UIR) bands (Gillett et al., 1973; Russell et al., 1977a; Willner et al., 1977), which are characterized by the main features at 3.3, 6.2, 7.7, 8.6 and $11.2 \mu \mathrm{ms}$, many minor bands and the underlying broad plateaus, many candidate materials of the origin have been proposed, such as hydrogenated amorphous carbon (Duley and Williams, 1981), quenched carbonaceous composite (Sakata et al., 1984) and polycyclic aromatic hydrocarbons (PAHs) (Léger and Puget, 1984; Allamandola et al., 1985). Since the UIR bands are observed in a wide variety of objects in various stages of stellar evolution, such as Herbig Ae/Be stars, HII regions, reflection nebulae, diffuse interstellar medium, post-asymptotic giant branch (AGB) stars and planetary nebulae (e.g. Russell et al., 1977b; Roche and Aitken, 1985; Cohen et al., 1986; Onaka et al., 1996), it appears that the carriers of the UIR bands should be stable materials. Furthermore, it is expected that the carriers are ubiquitous in space. Currently, PAHs are widely being used to examine the characteristics of UIR bands and are believed to be one of the most plausible origins of these bands.

PAHs consist of carbon atoms arranged in benzene rings terminated by hydrogen atoms. They have C-C and C-H bonds that exhibit vibrational modes in the mid-IR region. Since it has been reported that the UIR bands observed in

Copyright (C) The Society of Geomagnetism and Earth, Planetary and Space Sciences (SGEPSS); The Seismological Society of Japan; The Volcanological Society of Japan; The Geodetic Society of Japan; The Japanese Society for Planetary Sciences; TERRAPUB.

doi:10.5047/eps.2008.12.002 various objects show systematically different spectral features (Peeters et al., 2002; van Diedenhoven et al., 2004), the physical environments and chemical evolution in those kinds of objects can be derived from the details of band positions, relative intensities and widths of UIR bands through a series studies on PAHs. Recently, it was implied that part of the possible life cycle of PAHs depends on their distance from the central star, i.e., the ionized gas phase PAHs are dominant in the nearest region of the central star, then PAHs become neutral, and finally very small PAH grains, such as clusters of PAH molecules, become dominant at the distant region from the central star (Rapacioli et al., 2005). This also suggests a possible evolutional scenario of PAHs such that free-flying PAHs are formed from these PAH grains by photo-evaporation in photo-dissociation regions (Rapacioli et al., 2005, 2006).

In addition to these theoretical and observational studies, therefore, experiments on the laboratory synthesis of PAHs and their grains are necessary to understand the formation and subsequent growth into PAH grains and their alteration upon UV irradiation in various objects. Several laboratory IR spectra have been reported, for example, commercial PAHs embedded in KBr pellets (Bernas et al., 1966; Flickinger and Wdowiak, 1990), carbonaceous soot formed by burning benzene (Jäger et al., 2006), PAHs distributed in an inert matrix at low temperature (Szczepanski et al., 1992; Moutou et al., 1996; Hudgins and Sandford, 1998), gas phase (Flickinger et al., 1991; Colangeli et al., 1992; Joblin et al., 1995) and IR spectra of cationic PAHs (Hudgins et al., 1994; Hudgins and Allamandola, 1995; Oomens et al., 2003). However, the IR spectra of nanometer sized 
PAH grains have never been observed so far. It has been shown that nanometer-sized particles show significant phenomena, which cannot be expected based on the characteristics of the bulk material. For example, the melting point of gold decreases from 1336 to 1220 to $950 \mathrm{~K}$ as particle size decreases from macroscopic scale to 5 to $2-3 \mathrm{~nm}$ (Koga et al., 2004). In addition, the diffusion coefficient of copper atoms in gold nanoparticles changes to $8.3 \times 10^{-19}$ from $2.4 \times 10^{-28}\left(\mathrm{~m}^{2} \mathrm{~s}^{-1}\right)$ in the bulk material at $300 \mathrm{~K}$ (Mori et al., 1991). As a result, the time scale for forming nanoparticle alloys becomes markedly faster than that for the bulk material, i.e., from a couple of years to several seconds. Sometimes, even the stable crystal structures change for nanoparticles (Kimoto and Nishida, 1967). Actually, it has been reported that the crystal structure of pentacene is changed in thin film (Kakudate et al., 2007). Since it is nontrivial that PAH grains at the nanometer scale show similar properties with that of the macroscopic scale, we attempted to form nanometer sized PAHs grains and measured their IR spectra. Indeed, the importance of laboratory studies concerning optical spectra of nanometer sized solid particles has been suggested based on theoretical expectation. For example, the imaginary part in dielectric function becomes larger for small metallic grain compared with that of bulk. However, it is unclear how the optical properties of nanometer sized solid particles are different compared with that of bulk (Li, 2004). In this study, we performed the formation and growth of PAH grains at the nanometer scale, which are part of the life cycle of PAHs, and also performed alteration by UV irradiation, using mainly anthracene in the laboratory. This is the first report on IR spectra for nanometersized PAH grains.

\section{Experimental Procedures}

Anthracene has a molecular structure of three benzene rings in a straight alignment. It has two solo $\mathrm{C}-\mathrm{H}$ bonds and two quartet $\mathrm{C}-\mathrm{H}$ bonds, as shown in Fig. 1. The $\mathrm{C}-\mathrm{H}$ out-of-plane deformation mode exhibits bands in the 11$15 \mu \mathrm{m}$ region depending on the number of directly adjacent $\mathrm{H}$ atoms, because of the coupling between the vibrating $\mathrm{H}$ atoms bonded to the neighboring $\mathrm{C}$ atoms on the ring. Anthracene grains were produced by the gas evaporation method, which is also known as the smoke experiment and has been used to produce nanometer-sized metal, oxide or semiconductor particles (Kimoto et al., 1963; Kaito, 1978). The work chamber used was a glass cylinder $17 \mathrm{~cm}$ in inner diameter and $33 \mathrm{~cm}$ in height covered with a stainlesssteal plate with electrodes on its top and connected to a high-vacuum exhaust through a valve at its bottom. An evaporation source was set $3 \mathrm{~cm}$ above the heater in the chamber and a glass plate was set as a collector $5 \mathrm{~cm}$ above the evaporation source, as shown in Fig. 2. Commercial anthracene powder (Nacalai Tesque, Inc. 99\%) was charged on the upper molybdenum boat with a chromelalumel thermocouple. The chamber was evacuated down to $2.6 \times 10^{-4} \mathrm{~Pa}\left(2 \times 10^{-6}\right.$ Torr $)$ using a combination of a mechanical and a diffusion pump. After the valve was closed, He gas (99.99995\% up in purity) was introduced into the chamber up to $4.0 \times 10^{3} \mathrm{~Pa}$ (30 Torr), $1.1 \times 10^{4} \mathrm{~Pa}$ (80 Torr) or $2.0 \times 10^{4} \mathrm{~Pa}$ (150 Torr). Then, the lower molybdenum (a) anthracene

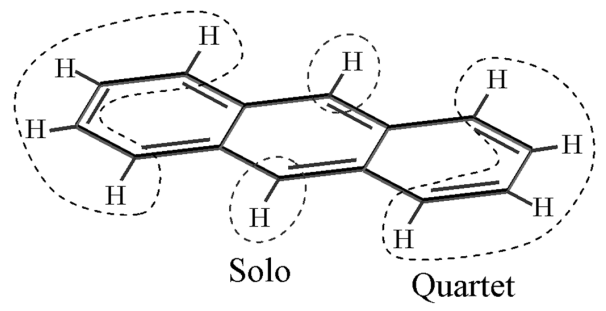

(b) diantracene

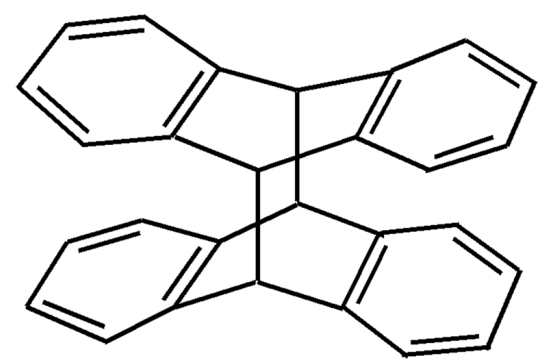

Fig. 1. Rough schematic models of (a) anthracene and (b) dianthracene molecules. The $\mathrm{C}-\mathrm{H}$ out-of-plane deformation mode exhibits bands in the 11-15 $\mu \mathrm{m}$ region depending on the number of directly adjacent $\mathrm{H}$ atoms with individual aromatic ring. Anthracene molecule has two solo $\mathrm{C}-\mathrm{H}$ bonds and two quartet $\mathrm{C}-\mathrm{H}$ bonds, which exhibit 11.31 and $13.77 \mu \mathrm{m}$ bands, respectively. Dianthracene is a photodimer of anthracene.

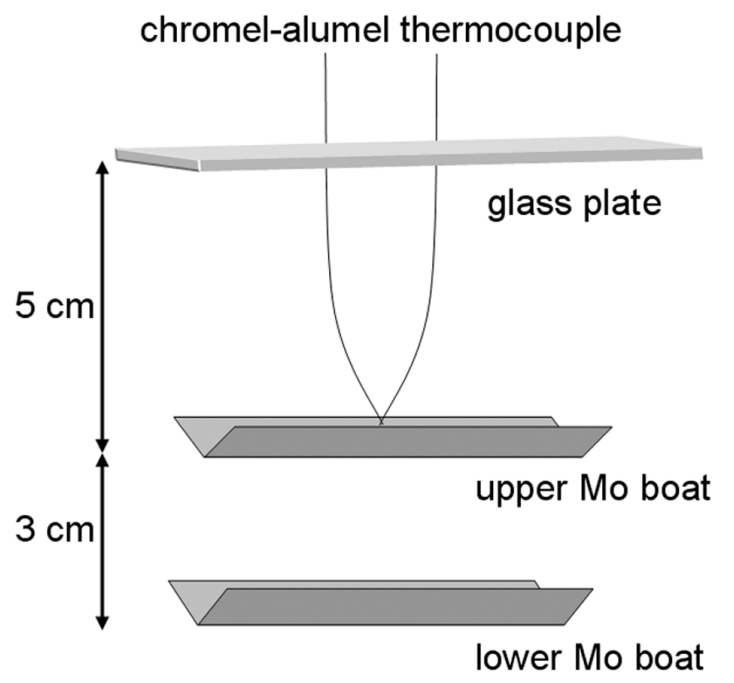

Fig. 2. Schematic image of apparatus used to produce PAH grains. Commercial PAH is evaporated from upper Mo boat by heating the lower Mo boat. PAH grains are produced from the gas phase as gas cools. TEM grids were set at the same position as the glass plate.

boat was resistively heated. As a result, the upper boat was heated at the rate of $10 \mathrm{~K} / \mathrm{s}$ by $\mathrm{He}$ gas convection and the temperature was controlled at $170^{\circ} \mathrm{C}$. The vapor pressure of anthracene at $170^{\circ} \mathrm{C}$ is approximately $6.7 \times 10^{2} \mathrm{~Pa}$ (5 Torr). The evaporated anthracene molecules were homogeneously nucleated as the gas cooled and their grains were formed in the smoke. The evaporated vapor subsequently cooled and became condensed in the gas atmosphere.

Coalescence growth is regarded as an important process 
in this method. Coalescence of the smoke particles has been explained as having two stages: a surface melting coalescence stage and a liquid-like coalescence stage (Kaito, 1978). These two stages depend mainly on grain size and temperature. The morphology and grain size are controlled by the mass density and temperature of the smoke, which depend on the atmospheric gas and gas pressure. The size of the particles also depends on their surface melting point. For example, metallic particles, such as iron or nickel with a diameter of 10-20 nm, can be produced by controlling the atmospheric gas pressure in the gas evaporation method. Since it has been suggested that the PAHs are formed in the outflows of carbon-rich AGB stars (Jura, 1987), the gas evaporation method roughly corresponds to the formation process of circumstellar PAH grains in the gas outflow from an evolved star. The produced grains are collected on a glass plate for the measurements of their IR spectra and on a standard $\mathrm{Cu}$ transmission electron microscope (TEM) grid supported with an amorphous carbon thin film for the observation of the size and the structure of the anthracene grains.

The mid-IR spectra, in the range from 2.5 to $25 \mu \mathrm{m}$, of the produced $\mathrm{PAH}$ grains embedded in a $\mathrm{KBr}$ pellet were measured with a Horiba FTIR-210 spectrometer. The wavelength resolution was $2 \mathrm{~cm}^{-1}$. The beam splitter was a Geevaporated $\mathrm{KBr}$ substrate, and the detector was deuteriated triglycine sulphate (DTGS). The UV-Vis spectra were measured with a Hitachi U-3300 spectrophotometer. The wavelength resolution was $0.5 \mathrm{~nm}$. The produced PAH grains were also observed using a Hitachi H-7100R TEM operated at an acceleration voltage of $100 \mathrm{kV}$.

\section{Synthesized PAH Grains}

A typical TEM image of anthracene grains produced in He gas of $1.1 \times 10^{4} \mathrm{~Pa}$ is shown in Fig. 3(a). The size of the anthracene grains was widely distributed from $5 \mathrm{~nm}$ to $5 \mu \mathrm{m}$. The electron diffraction (ED) pattern shows Bragg reflection spots attributed to anthracene crystal, i.e., anthracene molecules never broke during evaporation. The crystal structure of anthracene is monoclinic with the axes of $a=0.9463, b=0.6026$ and $c=0.8550 \mathrm{~nm}$ and angles of $\alpha=\gamma=90^{\circ}$ and $\beta=103.57^{\circ}$ (Mason, 1964). The ED pattern from the anthracene crystal was immediately reduced under electron beam irradiation of $10^{-3}-10^{-2} \mathrm{~A} / \mathrm{cm}^{2}$ (which is 10 times weaker than the typical intensity used in common observation) with acceleration voltage at $100 \mathrm{kV}$, and ultimately disappeared within about 60 seconds, i.e., the crystal structure of anthracene was easily broken. Although the crystal structure of anthracene grains is very sensitive to electron beam irradiation, the external geometry was retained after electron beam irradiation. During the TEM observation, the sublimation of anthracene grains was not observed.

With increasing He gas pressure, the size of the anthracene grains became slightly larger owing to the increase of the collision frequency with decreasing mean free path. Figure 4 shows the size distribution of anthracene grains. The mean sizes of the anthracene grains, which were determined in 1000 particles, produced in He gas of $4.0 \times 10^{3}$, $1.1 \times 10^{4}$ and $2.0 \times 10^{4} \mathrm{~Pa}$ were $37.7,49.6$ and $58.2 \mathrm{~nm}$, respectively. The number of carbon atoms is of the order of

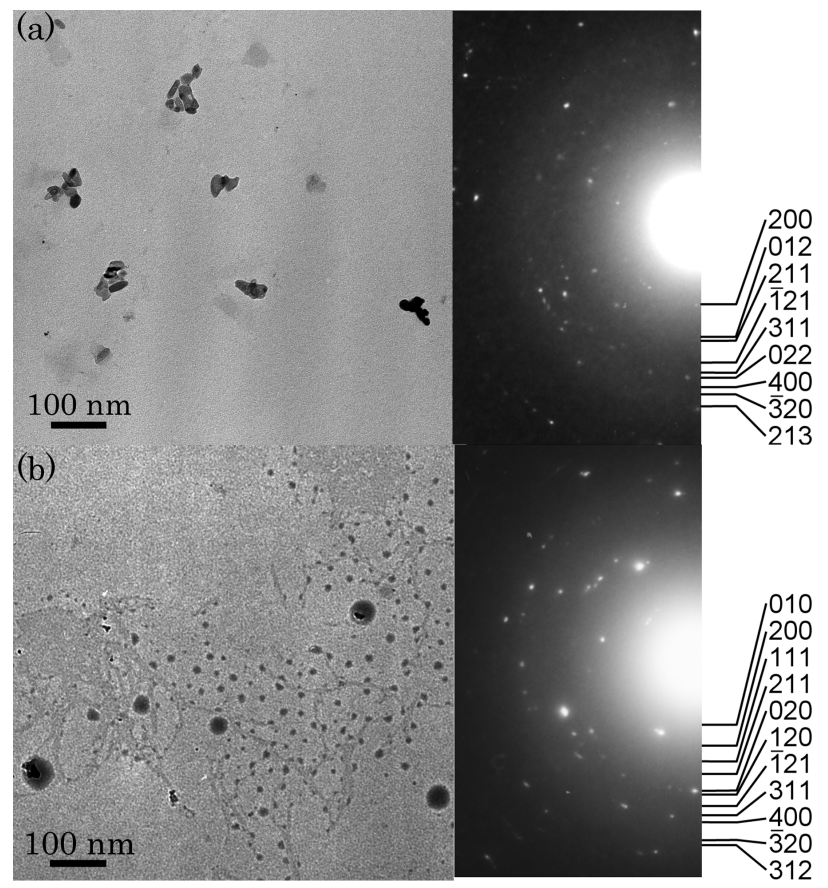

Fig. 3. Typical TEM images and corresponding ED patterns of anthracene grains produced in $1.1 \times 10^{4} \mathrm{~Pa} \mathrm{He}$ gas. (a) The upper boat was heated at the rate of $10 \mathrm{~K} / \mathrm{s}$ by He gas convection and the temperature was controlled at $170^{\circ} \mathrm{C}$. The mean diameter of anthracene grains was $49.6 \mathrm{~nm}$, which corresponds to the carbon number of $1.9 \times 10^{6}$ if the grains are hemispherical. (b) Temperature of the evaporation source was raised at a slower rate of $2.5 \mathrm{~K} / \mathrm{s}$ and the temperature was controlled at $170^{\circ} \mathrm{C}$. In addition to the anthracene grains, neuron-like anthracene films were formed on the amorphous carbon substrate. These TEM images were taken using the Hitachi H-7100R TEM operated at an accelerating voltage of $100 \mathrm{kV}$.

$10^{5}-10^{6}$. Occasionally, significantly larger grains with micrometer diameter were seen.

When the temperature of the evaporation source was raised at a slower rate of $2.5 \mathrm{~K} / \mathrm{s}$, neuron-like anthracene films were formed on the amorphous carbon substrate along with anthracene grains, as shown in Fig. 3(b). Since the anthracene grains were also produced, the anthracene films would be formed from anthracene evaporated at a lower temperature. Because the amount of evaporant is less at lower temperatures, the anthracene grains produced in the smoke are small. Therefore, the small anthracene grains might easily migrate on the carbon substrate and form anthracene films by coalescence. The specimen including anthracene film shows the characteristic $12 \mu \mathrm{m}$ band, as described in Section 4.

\section{Infrared Spectra of Anthracene Grains}

The anthracene grains produced were embedded in $\mathrm{KBr}$ pellets and their mid-IR spectra were measured. The IR spectra of anthracene grains produced in $\mathrm{He}$ gas of $4.0 \times 10^{3}, 1.1 \times 10^{4}$ and $2.0 \times 10^{4} \mathrm{~Pa}$ showed many significant features including 11.31 - and $13.77-\mu \mathrm{m}$ bands, which are attributed to the vibrations of aromatic solo and quartet $\mathrm{C}-\mathrm{H}$ out-of-plane bending modes, respectively. The typical IR spectrum of anthracene grains with a mean diameter of $49.6 \mathrm{~nm}$ produced in $1.1 \times 10^{4} \mathrm{~Pa} \mathrm{He}$ gas, corresponding to the anthracene grains in Fig. 3(a) is 


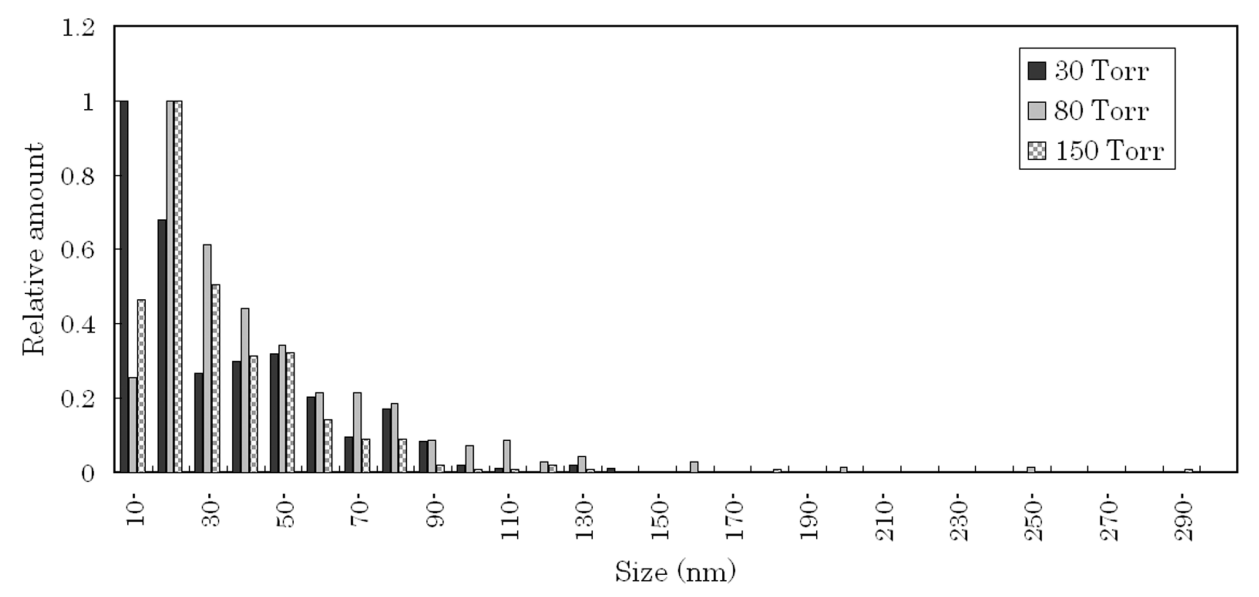

Fig. 4. Size distribution of nanometer-sized anthracene grains produced by the gas evaporation method. The mean sizes of the grains produced in He gas of $4.0 \times 10^{3}, 1.1 \times 10^{4}$ and $2.0 \times 10^{4}$ Pa were $37.7,49.6$ and $58.2 \mathrm{~nm}$, respectively.

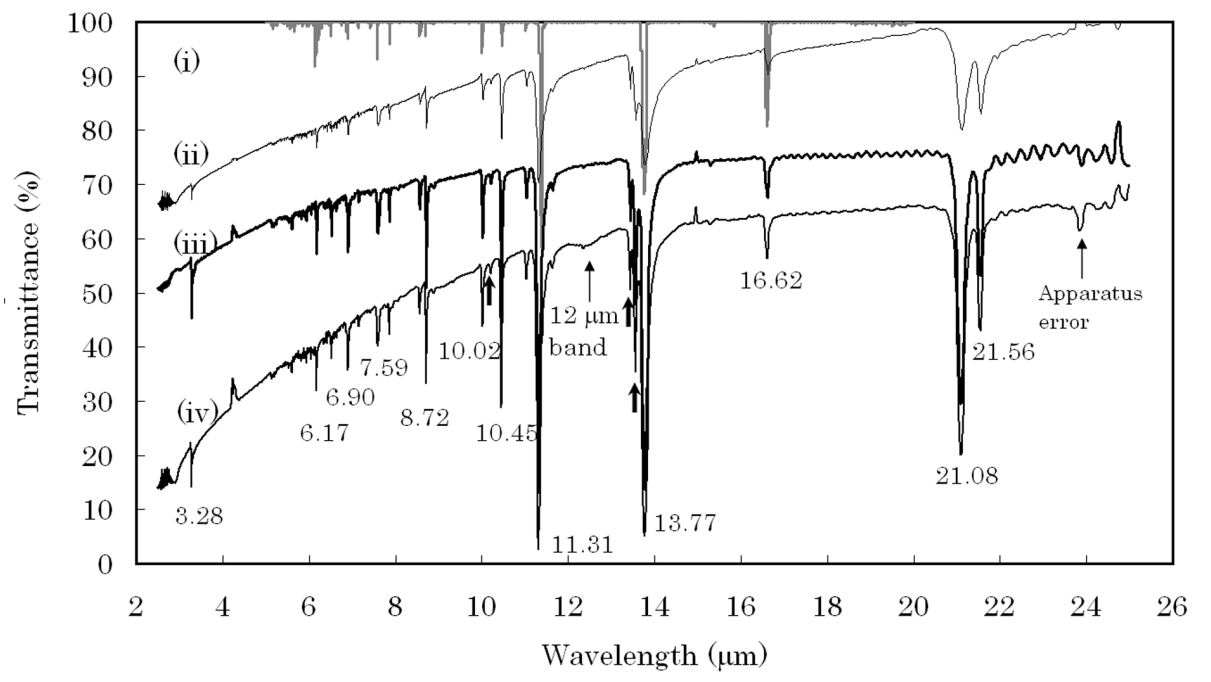

Fig. 5. Mid-IR spectra corresponding to (i) molecule in Ar matrix, (ii) commercial powder of anthracene in KBr pellet, (iii) laboratory-produced grains shown in Fig. 2(a) and (iv) laboratory-produced films and grains shown in Fig. 2(b). The IR spectrum of molecule (i) was extracted from the on-line database of IR spectra of PAHs by Hudgins et al. on the Astrochemistry Laboratory website (http://www-space.arc.nasa.gov/ astrochm/pahdata/) and was modified. It has not been recorded at wavelengths longer than $20 \mu \mathrm{m}$. A significant $12 \mu \mathrm{m}$ band appeared in the spectrum of anthracene grains. It corresponds to the plateau feature in astronomically observed spectra. IR spectra (ii) and (iii) of the samples embedded in KBr pellets were measured with a Fourier-transform infrared spectrometer (Horiba Inc. FT210). The wavelength resolution was $2 \mathrm{~cm}^{-1}$. The spectra were arbitrarily-positioned for clarity in the direction of longitudinal axis.

shown in Fig. 5(iii). Characteristic features are coincident with the spectrum measured for anthracene molecules dispersed in an Ar matrix at $10 \mathrm{~K}$ (Hudgins and Sandford, 1998). The IR spectrum, ranging from 5 to $20 \mu \mathrm{m}$, of the molecule was taken from the following webpage (http://www-space.arc.nasa.gov/ astrochm/pahdata/) and modified, as shown in Fig. 5(i). The characteristic features and most plausible candidates of their origin are listed in Table 1. Some differences in the full width of halfmaximum, peak intensities and positions between IR spectra of grains and molecules are visible. In particular, the IR spectra of the grains and the commercial powder have an extra peak at $10.21 \mu \mathrm{m}$ and two additional peaks at 13.43 and $13.56 \mu \mathrm{ms}$ along with the peak at $13.77 \mu \mathrm{m}$ compared with the spectra of matrix isolated molecules as indicated by arrows in Fig. 5(iv). In order to see the influence of impurities, a shutter was prepared just below the glass plate. As a result, impurities with sublimation temperatures lower than anthracene could be eliminated. However, the IR spectral features did not change. In addition, although the commercial anthracene powder of the evaporant remained during the experiment, the change of the IR spectra was negligible, i.e., there is no influence of impurities with higher sublimation temperature. Therefore, the bands produced are attributed to new transition bands caused by the aggregation of anthracene molecules independent with crystalline size.

In the spectrum of anthracene films and grains corresponding to Fig. 3(b), a 12- $\mu \mathrm{m}$ band newly appears compared with that of anthracene grains, which is shown in Fig. 5(iv). The 12- $\mu \mathrm{m}$ band has similarly appeared in the spectra of anthracene grains produced with lower heating rates in $4.0 \times 10^{3}$ and $2.0 \times 10^{4} \mathrm{~Pa} \mathrm{He}$ gas. Although the $12-\mu \mathrm{m}$ band is very weak, it significantly shows up in the spectra of condensed anthracene. Previously, although ex- 
Table 1. Absorption peak positions $(\mu \mathrm{m})$ of each spectrum and known assignments.

\begin{tabular}{|c|c|c|c|c|c|c|}
\hline $\begin{array}{l}\text { Molecules } \\
\text { Fig. 3(i) }\end{array}$ & $\begin{array}{l}\text { Cationic } \\
\text { molecules }^{\mathrm{a}}\end{array}$ & $\begin{array}{l}\text { Dianthra- } \\
\text { cene }^{\mathrm{b}}\end{array}$ & $\begin{array}{l}\text { Bulk } \\
\text { Fig. 3(ii) }\end{array}$ & $\begin{array}{l}\text { Grains } \\
\text { Fig. 3(iii) }\end{array}$ & $\begin{array}{l}\text { UV-irradiated grains } \\
\text { (viii) in Fig. 5(b) }\end{array}$ & Assignments $^{\mathrm{c}}$ \\
\hline \multicolumn{7}{|l|}{3.19} \\
\hline \multicolumn{7}{|l|}{3.20} \\
\hline \multicolumn{7}{|l|}{3.22} \\
\hline 3.26 & & 3.27 & 3.28 & 3.28 & 3.26 & \\
\hline 3.30 & & 3.29 & & & 3.29 & 3.3 aromatic $\mathrm{C}-\mathrm{H}$ stretching mode \\
\hline 3.31 & & 3.32 & & & 3.32 & \\
\hline \multirow[t]{3}{*}{3.36} & & 3.33 & & & 3.33 & \\
\hline & & 3.40 & & & 3.39 & 3.4 aliphatic $\mathrm{C}-\mathrm{H}$ stretching mode in methyl groups \\
\hline & & 3.42 & & & 3.42 & $\begin{array}{l}\text { C-H stretching mode in hydrogenated PAHs } \\
\text { hot band of the aromatic C-H stretch }\end{array}$ \\
\hline 5.21 & & & & & & 5.2 combination mode, $\mathrm{C}-\mathrm{H}$ bend and $\mathrm{C}-\mathrm{C}$ stretch \\
\hline \multicolumn{7}{|l|}{5.25} \\
\hline \multicolumn{7}{|l|}{5.42} \\
\hline \multicolumn{7}{|l|}{5.51} \\
\hline \multicolumn{7}{|l|}{5.57} \\
\hline \multicolumn{7}{|l|}{5.62} \\
\hline 5.66 & & & & & & 5.65 combination mode, $\mathrm{C}-\mathrm{H}$ bend and $\mathrm{C}-\mathrm{C}$ stretch \\
\hline \multicolumn{7}{|l|}{5.75} \\
\hline \multicolumn{7}{|l|}{5.86} \\
\hline 5.97 & & & & & & 6.0 C-O stretching mode (?) \\
\hline 6.14 & & & 6.17 & 6.17 & & \\
\hline \multirow[t]{2}{*}{6.21} & & & & & & 6.2 aromatic $\mathrm{C}-\mathrm{C}$ stretching mode \\
\hline & 6.30 & & & & & \\
\hline 6.49 & 6.49 & & 6.52 & 6.52 & & \\
\hline 6.85 & & 6.80 & & & 6.79 & \\
\hline \multirow[t]{7}{*}{6.89} & 6.87 & 6.88 & 6.90 & 6.90 & 6.87 & 6.9 aliphatic $\mathrm{C}-\mathrm{H}$ bending modes \\
\hline & 6.99 & & & & & \\
\hline & 7.05 & & & & & \\
\hline & 7.09 & & & & & \\
\hline & 7.11 & & & 7.15 & & \\
\hline & 7.33 & & & & & \\
\hline & 7.39 & & & & & \\
\hline 7.43 & 7.46 & & & & & \\
\hline 7.59 & 7.61 & & 7.59 & 7.59 & & 7.6 $\mathrm{C}-\mathrm{C}$ stretching and $\mathrm{C}-\mathrm{H}$ in-plane bending modes \\
\hline \multirow[t]{4}{*}{7.86} & 7.75 & 7.74 & 7.86 & 7.86 & 7.75 & 7.8 $\mathrm{C}-\mathrm{C}$ stretching and $\mathrm{C}-\mathrm{H}$ in-plane bending modes \\
\hline & & 8.20 & & & 8.20 & \\
\hline & 8.41 & & & & & \\
\hline & 8.45 & & & & & \\
\hline 8.57 & & 8.62 & 8.57 & 8.57 & 8.62 & 8.6 C-H in-plane bending modes \\
\hline \multirow[t]{4}{*}{8.70} & & & 8.72 & 8.72 & & \\
\hline & & & 8.88 & 8.88 & & \\
\hline & & 9.07 & & & 9.07 & \\
\hline & & 9.71 & & & 9.70 & \\
\hline \multirow[t]{2}{*}{9.99} & & & 10.02 & 10.02 & & \\
\hline & & & 10.21 & 10.21 & & \\
\hline \multirow[t]{2}{*}{10.47} & & 10.59 & 10.45 & 10.45 & 10.59 & \\
\hline & & 10.65 & & & & \\
\hline 11.03 & 10.96 & & 11.03 & 11.03 & & 11.0 C-H out-of-plane bending modes, solo, cation \\
\hline \multirow[t]{7}{*}{11.39} & & & 11.31 & 11.31 & & 11.2 C-H out-of-plane bending modes, solo, neutral \\
\hline & & & 11.64 & 11.64 & & \\
\hline & & 12.27 & & & 12.27 & \\
\hline & & & & 12.44 & & 12.7 C-H out-of-plane bending modes, trio, cation (?) \\
\hline & & 13.12 & & & 13.08 & \\
\hline & 13.36 & 13.28 & 13.43 & 13.43 & 13.25 & \\
\hline & & & 13.56 & 13.56 & & 13.6 C-H out-of-plane bending modes, quartet \\
\hline
\end{tabular}


Table 1. (continued).

\begin{tabular}{|c|c|c|c|c|c|c|}
\hline $\begin{array}{l}\text { Molecules } \\
\text { Fig. 3(i) }\end{array}$ & $\begin{array}{l}\text { Cationic } \\
\text { molecules }^{\text {a }}\end{array}$ & $\begin{array}{l}\text { Dianthra- } \\
\text { cene }^{\text {b }}\end{array}$ & $\begin{array}{l}\text { Bulk } \\
\text { Fig. 3(ii) }\end{array}$ & $\begin{array}{l}\text { Grains } \\
\text { Fig. 3(iii) }\end{array}$ & $\begin{array}{l}\text { UV-irradiated grains } \\
\text { (viii) in Fig. } 5 \text { (b) }\end{array}$ & Assignments $^{c}$ \\
\hline \multirow[t]{2}{*}{13.78} & & & 13.77 & 13.77 & & \\
\hline & & 14.78 & & & 14.69 & 14.2 C-H out-of-plane bending modes, quartet \\
\hline \multirow[t]{4}{*}{16.59} & & 16.69 & 16.62 & 16.62 & 16.73 & $\begin{array}{l}16.4 \text { in-plane and out-of-plane } \mathrm{C}-\mathrm{C}-\mathrm{C} \text { bending modes } \\
\text { in pendant ring (?) }\end{array}$ \\
\hline & & 21.10 & 21.08 & 21.08 & 21.08 & Unknown \\
\hline & & & 21.56 & 21.56 & & Unknown \\
\hline & & 23.00 & & & 22.94 & \\
\hline
\end{tabular}

${ }^{a}$ Hudgins et al. (http://www-space.arc.nasa.gov/ astrochm/pahdata/).

${ }^{\mathrm{b}}$ Singh and Sandoefy (1969).

${ }^{\mathrm{c}}$ Tielens (2005).

istence of a feature was calculated at $12.3 \mu \mathrm{m}$ for longaxis polarization, it was not found in solution spectra and in stretched polyethylene spectra, and finally it was not assigned (Radziszewski and Michl, 1985). Since the 12- $\mu \mathrm{m}$ band has never appeared in the spectra of molecules and commercial bulk powder, nor in that of anthracene grains excluding film, we considered that the $12-\mu \mathrm{m}$ band was caused by the anthracene film. There are other two possible origins of the $12-\mu \mathrm{m}$ band: the duo or trio C-H out-ofplane bending mode and group vibrations of $(\mathrm{CH})_{n}(n=2$ or 3). However, since the features, such as relative intensity or peak position, of the $12-\mu \mathrm{m}$ band remained the same in all experimental runs with various He gas pressures, we believe that the origin of the $12-\mu \mathrm{m}$ band is anthracene itself.

\section{Stability on UV Irradiation}

It has been suggested that when the PAHs consisting of 25 carbon atoms form grains via van der Waals forces, the PAH grains are easily broken by far-UV photon absorption (Léger and Puget, 1984; Tielens et al., 1999). It has also been suggested that the PAH grains are photo-evaporated, which produces free-flying PAHs in photo-dissociation regions (Rapacioli et al., 2005; Sakon et al., 2007). Anthracene has roughly two absorption features at 210-290 and $340-410 \mathrm{~nm}$ in the range of 200 to $800 \mathrm{~nm}$, as shown in Fig. 6. In order to elucidate the stability of anthracene grains to UV irradiation, the anthracene grains, which were produced in $1.1 \times 10^{4} \mathrm{~Pa}$ of $\mathrm{He}$ gas and embedded in a $\mathrm{KBr}$ pellet, corresponding to Fig. 3(a), were irradiated for 1100 hours with UV radiation at 254 and $365 \mathrm{~nm}$. The UV lamps at 254 and $365 \mathrm{~nm}$ have a width of about $20 \mathrm{~nm}$. The two different irradiation wavelengths correspond to the central wavelength of the absorption features. The intensities at 254 and $365 \mathrm{~nm}$ of the UV lamp are 61 and $74 \mathrm{~mW} / \mathrm{cm}^{2}$, respectively. The absorption efficiencies at 254 and $365 \mathrm{~nm}$ regions are similar. In the case of irradiation for 15 hours, the total fluxes at 254 and $365 \mathrm{~nm}$ are $4.2 \times 10^{21}$ and $7.4 \times 10^{21}$ photons $/ \mathrm{cm}^{2}$, respectively.

In the case of irradiation at $254 \mathrm{~nm}$, almost all features shown in the spectrum for 0 hour in Fig. 7(a), such as peaks at $6.17,6.90,7.59,8.72,10.02,10.45,11.31,13.77,21.12$ and $21.56 \mu \mathrm{m}$, gradually decreased with increasing irradiation time. In contrast, new bands appeared at 8.19, 8.62, $9.70,10.59,12.27$ and $14.69 \mu \mathrm{m}$, and the band at $16.62 \mu \mathrm{m}$ seemed to be shifted to $16.72 \mu \mathrm{m}$ and appeared with higher intensity. Then, the feature at $254 \mathrm{~nm}$ disappeared and the feature at $365 \mathrm{~nm}$ decreased, as shown in Fig. 6(a). In the case of irradiation at $365 \mathrm{~nm}$, the peaks in the mid-infrared region were changed similarly to the case of $254 \mathrm{~nm}$ irradiation, although the change was rapid compared with the case of $254 \mathrm{~nm}$, as indicated in Fig. 7(b). The features completely disappeared within 15 hours upon irradiation at $365 \mathrm{~nm}$, although initial bands remained after 100 hours of $254 \mathrm{~nm}$ irradiation. At this time, the absorption features at $254 \mathrm{~nm}$ also disappeared, as did the absorption feature corresponding to the irradiated wavelength at $365 \mathrm{~nm}$, as shown in Fig. 6(b). Namely, irradiation at $365 \mathrm{~nm}$ had a greater effect on anthracene grains than that at $254 \mathrm{~nm}$ in spite of its weaker energy. This result implies that the alteration of PAHs by UV irradiation depends on the wavelength. Photon fluxes for the disappearance of half-values of the features were $2.25 \times 10^{22}$ and $2.02 \times 10^{21}$ photons $/ \mathrm{cm}^{2}$ at 254 and $365 \mathrm{~nm}$, respectively. The value of the appearance features doubled upon irradiation at similar photon fluxes. The decreasing rates of the absorption intensity at the $11.3 \mu \mathrm{m}$ feature under irradiation of both wavelengths are shown in Fig. 8. The photon fluxes in various environments have been reported as $3.4 \times 10^{12}$ photons $\mathrm{cm}^{-2} \mathrm{~s}^{-1}$ in a reflection nebula NGC 7023, which is illuminated by a young star (Rogers et al., 1995), $1.4 \times 10^{3}$ and $4.8 \times 10^{3}$ photons $\mathrm{cm}^{-2} \mathrm{~s}^{-1}$ in a dense molecular cloud (Prasad and Tarafdar, 1983; Mennella et al., 2003), $\sim 10^{8}$ photons $\mathrm{cm}^{-2}$ $\mathrm{s}^{-1}$ at the edge of such a cloud (Mathis et al., 1983) and $6.4 \times 10^{9}$ photons $\mathrm{cm}^{-2} \mathrm{~s}^{-1}$ at a heliocentric distance of 5 AU, which corresponds to the distance between Jupiter and the Sun, from the coronal photon flux of the young Sun (Dorren et al., 1995). The experimental photon flux for the disappearance of half-values of the features under 365 $\mathrm{nm}$ corresponds to that for $1.9 \times 10^{1}, 1.3-4.4 \times 10^{10}, \sim 10^{5}$ and $9.8 \times 10^{3}$ year, respectively. This rough estimation suggests that although the spectral alteration of anthracene does not appear around dense molecular clouds, it may occur in reflection nebula and young stellar environments if anthracene grains survive into that stage.

The disappearance of UV-Vis features shown in Fig. 6(b) implies that the band gaps of anthracene were varied by UV irradiation. In addition, since the infrared features drastically changed, the $\mathrm{C}-\mathrm{H}$ bonding of anthracene molecules 

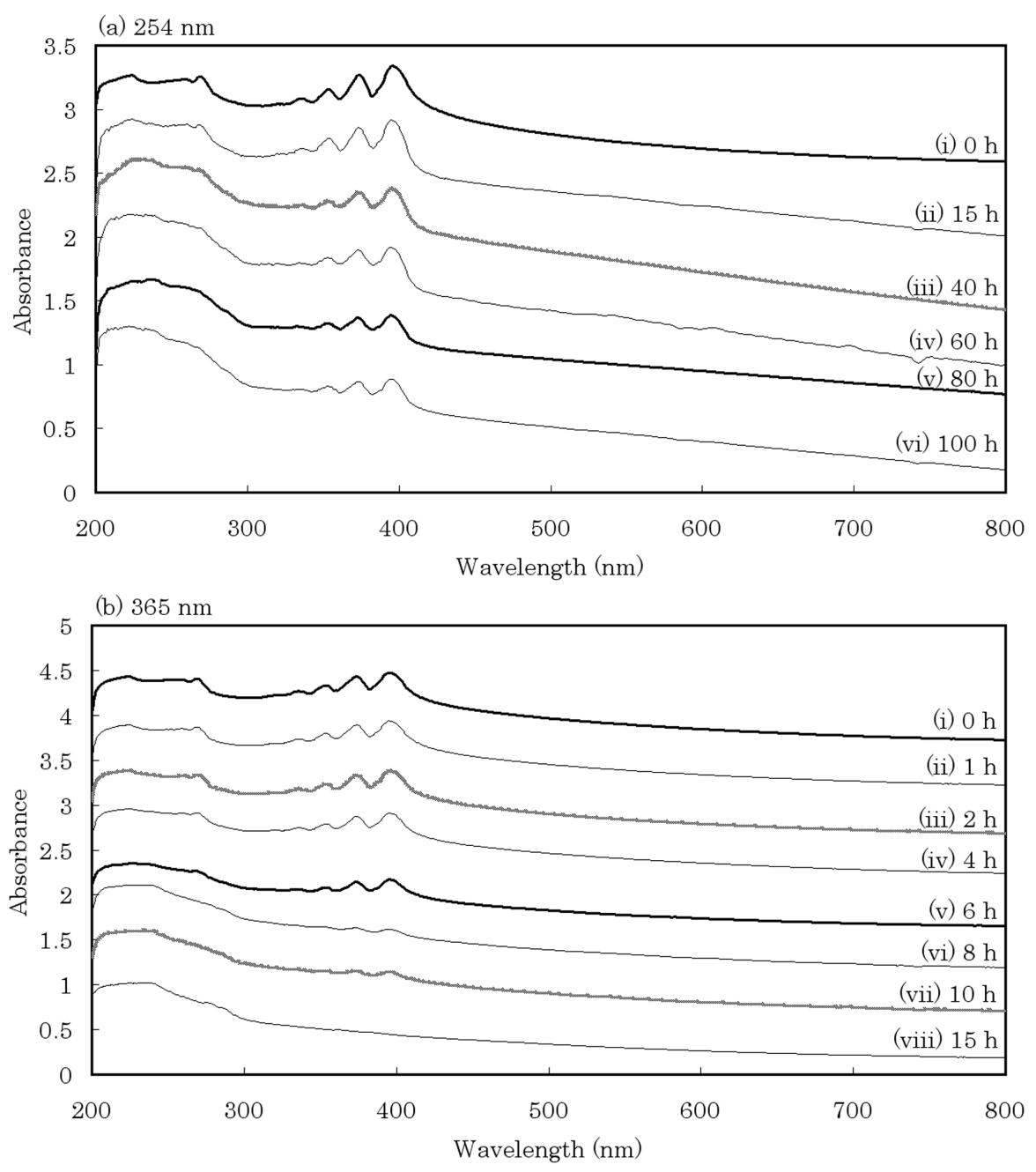

Fig. 6. Spectral change in UV-Vis spectra of anthracene grains produced in $1.1 \times 10^{4} \mathrm{~Pa} \mathrm{He}$ gas embedded in $\mathrm{KBr}$ pellets after UV irradiation for 1-100 hours at (a) 254 and (b) $365 \mathrm{~nm}$. The intensities at 254 and $365 \mathrm{~nm}$ of the UV lamp are 61 and $74 \mathrm{~mW} / \mathrm{cm}^{2}$, respectively. The total fluxes at $254 \mathrm{~nm}$ are $4.2 \times 10^{21}, 1.1 \times 10^{22}, 1.7 \times 10^{22}, 2.8 \times 10^{22}$ and $8.4 \times 10^{22}$ photons $/ \mathrm{cm}^{2}$, for $15,40,60,80$ and 100 hours, respectively. The total fluxes at $365 \mathrm{~nm}$ are $5.0 \times 10^{20}, 1.0 \times 10^{21}, 2.0 \times 10^{21}, 3.0 \times 10^{21}, 4.0 \times 10^{21}, 5.0 \times 10^{21}$ and $7.6 \times 10^{21}$ photons $/ \mathrm{cm}^{2}$ for $1,2,4,6,8,10$ and 15 hours, respectively. Anthracene has roughly two absorption features at $210-290$ and 310-440 nm in the range of 200 to $800 \mathrm{~nm}$, as seen in spectra (i). (a) The feature at $210-290 \mathrm{~nm}$ gradually disappeared after UV irradiation at $254 \mathrm{~nm}$. The feature at $310-440 \mathrm{~nm}$ still remained after as long as 100 hour. (b) Both features immediately and simultaneously disappeared after UV irradiation at $365 \mathrm{~nm}$. The spectra were arbitrarily-positioned for clarity in the direction of longitudinal axis.

should be altered. After several studies, we concluded that the new features are caused by dianthracene, i.e., a photodimer of anthracene was produced (Fig. 1). The features have been compared with those of a previously reported spectrum in Table 1. It is known that dianthracene reverts to anthracene upon UV irradiation at $300 \mathrm{~nm}$ or shorter. Nevertheless, dianthracene was formed by irradiation at $254 \mathrm{~nm}$ in this experiment. This might be the reason behind the slower change of the spectral features by UV irradiation at $254 \mathrm{~nm}$ compared to $365 \mathrm{~nm}$.

In order to elucidate the conditions of spectral change, several experiments were performed. The spectrum change was not observed when anthracene, before being embedded in $\mathrm{KBr}$, was irradiated with UV in $\mathrm{He}$ of $4.0 \times 10^{4} \mathrm{~Pa}$ (300 Torr). In the case of irradiation in air, the spectral features did not change, although the color of anthracene became yellowish. It has been observed that yellow anthraquinone is formed by irradiation in the presence of oxygen. Nevertheless, the spectrum of the yellowish sample does not correspond with anthraquinone. The spectral evolution was completely duplicated with the irradiation of anthracene embedded in $\mathrm{KCl}$ or $\mathrm{NaCl}$. Namely, potassium and bromide do not affect the formation of dianthracene. When anthracene and $\mathrm{KBr}$ were mixed and irradiated with UV before being made into a pellet under pressure, the spectral change was negligible. Accordingly, the pressure in forming the pellet is a clue to the spectral evolution. To form a $\mathrm{KBr}$ pellet, the mixture of anthracene and $\mathrm{KBr}$ powders was pressed at $6 \times 10^{3} \mathrm{~kg} / \mathrm{cm}^{2}$. It is known that the formation of dianthracene is accelerated depending on the domain structure of anthracene crystals (Ramdas et al., 1980). Actually, formation of dianthracene in $\mathrm{KBr}$ pellets has been previously reported using bulk anthracene crystals with similar trends even in nanometer sized anthracene grains (Bernas et al., 1966; Chandross and Ferguson, 1966). Since the crystal structure of anthracene may be fragile because it is a molecular crystal, pressing will reduce the crystallinity of anthracene grains. However, the absorption features at- 

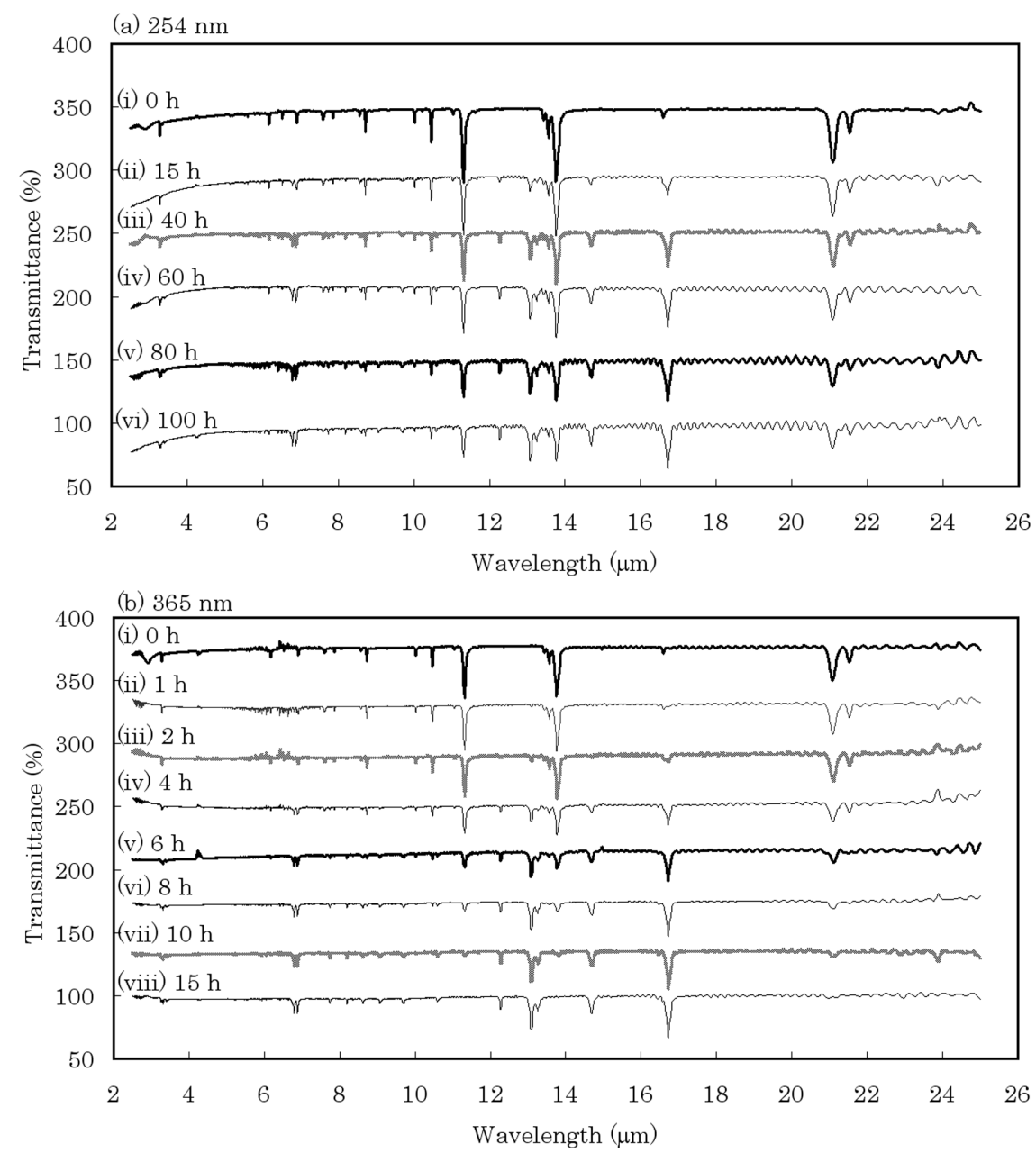

Fig. 7. Mid-IR spectra of anthracene grains produced in $1.1 \times 10^{4} \mathrm{~Pa} \mathrm{He}$ gas. Spectral change by UV irradiation at (a) $254 \mathrm{~nm}$ and (b) $365 \mathrm{~nm}$ is shown for 1-100 hours. The intensities at 254 and $365 \mathrm{~nm}$ of the UV lamp are 61 and $74 \mathrm{~mW} / \mathrm{cm}^{2}$, respectively. The total fluxes at $254 \mathrm{~nm}$ are $4.2 \times 10^{21}, 1.1 \times 10^{22}, 1.7 \times 10^{22}, 2.8 \times 10^{22}$ and $8.4 \times 10^{22}$ photons $/ \mathrm{cm}^{2}$, for $15,40,60,80$ and 100 hours, respectively. The total fluxes at $365 \mathrm{~nm}$ are $5.0 \times 10^{20}, 1.0 \times 10^{21}, 2.0 \times 10^{21}, 3.0 \times 10^{21}, 4.0 \times 10^{21}, 5.0 \times 10^{21}$ and $7.6 \times 10^{21}$ photons $/ \mathrm{cm}^{2}$ for $1,2,4,6,8,10$ and 15 hours, respectively. (a) In the case of irradiation at $254 \mathrm{~nm}$, the spectral change gradually takes place with increasing irradiation time. (b) In contrast, in the case of irradiation at $365 \mathrm{~nm}$, the spectral change was similarly to that at $254 \mathrm{~nm}$ irradiation but the change was very fast compared with that at $254 \mathrm{~nm}$. Photon fluxes for the disappearance of the half-value of the features were $2.25 \times 10^{22}$ and $2.02 \times 10^{21}$ photons $/ \mathrm{cm}^{2}$ at 254 and $365 \mathrm{~nm}$, respectively. The value of the appearance features became twice upon irradiation at similar photon fluxes. The spectra were arbitrarily-positioned for clarity in the direction of longitudinal axis.

(a)

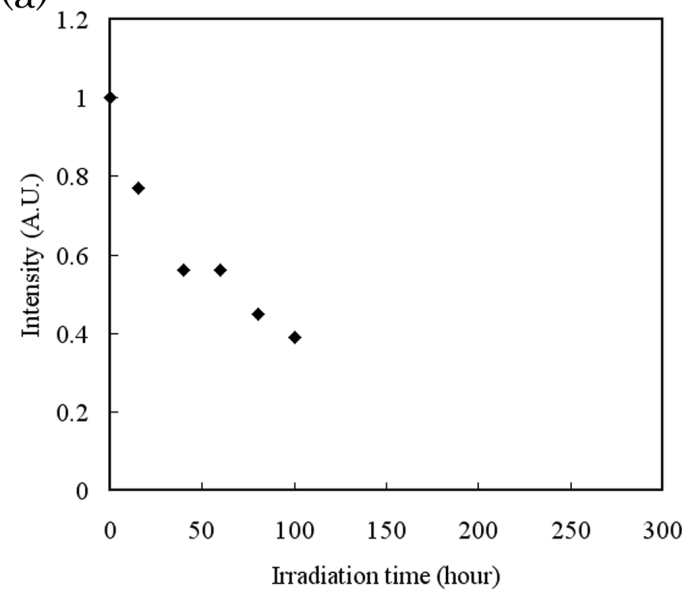

(b)

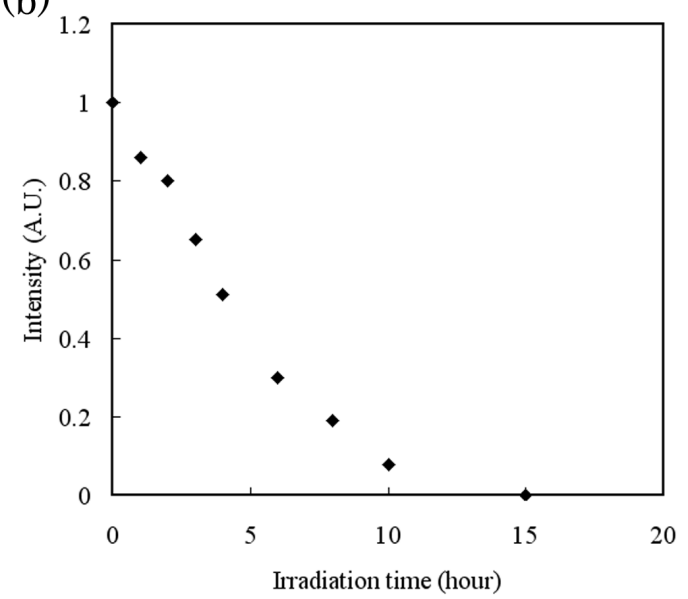

Fig. 8. The correlation between the absorption intensity at the $11.3 \mu \mathrm{m}$ feature and the irradiation time under (a) 254 and (b) $365 \mathrm{~nm}$, respectively. The times and total photon fluxes at the half-value of the intensity at the $11.3 \mu \mathrm{m}$ feature are 80 and 4 hours and $2.25 \times 10^{22}$ and $2.02 \times 10^{21}$ photons $/ \mathrm{cm}^{2}$, respectively. 
tributed to the anthracene molecule were not changed after it was embedded in the $\mathrm{KBr}$ pellet. Similar spectral changes were observed even when the pressure for pressing the $\mathrm{KBr}$ pellet was as low as $7.5 \times 10^{2} \mathrm{~kg} / \mathrm{cm}^{2}$. When a similar irradiation experiment was performed on other PAHs, such as phenanthrene, naphthacene (tetracene), pentacene, pyrene, chrysene and coronene, no alteration of the spectral features was observed except for anthracene, even when xenon white light was irradiated.

\section{Astronomical Implications}

Our experimental result concerning the $12 \mu \mathrm{m}$ band caused by anthracene films implied that if anthracene and possibly other PAHs deposited on the previously condensed solid grains, such as silicon carbide, graphitic carbon, iron or possibly oxide, PAHs would grow on the grain in a twodimensional structure and form PAH films. Then, the surface PAH layer might be the cause of the plateau feature at $11-15 \mu \mathrm{m}$. Indeed, silicon carbide grains with PAHs on their surface have been found in a carbonaceous meteorite (Frenklach et al., 1989). Two-dimensional PAHs can also exist in the gas phase. The PAH surface layer will later be coated by an ice layer in the cooler region. If those particles are transferred to a molecular cloud, photodimers should be produced in the carbonaceous layer between the core and the surface ice layer. In the case of freeflying PAH molecules, they emit after excitation by absorption of single UV photons. Since nanometer sized grains have a larger heat capacity than $\mathrm{PAH}$ molecules, the grains have to be heated by other source or photodimers have to be released from the grain surface for efficient emission from photodimers. In that case, the infrared spectrum will become broader, because many peaks appear in the midinfrared region. In addition to anthracene, it is known that naphthacene and coronene can form dimers. Therefore, although infrared spectra of neutral, anionic and cationic PAHs were measured actively, it is necessary to obtain the infrared spectrum of PAH dimers, because UV rays are present ubiquitously in space. Our experimental results suggest the possibility that the disappearance of UIR bands in the HII region is a result of the broadness of infrared features caused by the formation of photodimers.

\section{Summary}

Anthracene grains with 35-60 nm mean diameter were produced by evaporating commercial anthracene powder in He gas. The anthracene grains showed somewhat different features concerning width, relative intensities and positions from anthracene molecules. In addition, the $12-\mu \mathrm{m}$ band, which has never appeared in the spectra of molecular and commercial powder of anthracene, was observed from the anthracene films. After UV irradiation, the characteristic mid-IR features of the anthracene grains were dramatically changed owing to the formation of anthracene photodimers, i.e., dianthracene, depending on the irradiated wavelength. Currently, similar experiments are being performed in order to understand the growth mechanisms and the cause of the significant optical properties of PAHs and their grains using other PAHs, such as naphthacene, naphthalene, pyrene, chrysene and coronene.
Acknowledgments. This work was supported in part by a Grant-in-Aid for Young Scientists (start-up) from the KAKENHI (19840048) of JSPS.

\section{References}

Allamandola, L. J., A. G. G. M. Tielens, and J. R. Barker, Polycyclic aromatic hydrocarbons and the unidentified infrared emission bands: Auto exhaust along the milky way!, Astrophys. J., 290, L25-L28, 1985.

Bernas, A., D. Leonardi, and M. Renaud, On photoreactions of anthracene in solid matrix-I. photodimerization, Photochem. Photbiol., 5, 721-731, 1966.

Chandross, E. A. and J. Ferguson, Photodimerization of crystalline anthracene. The photolytic dissociation of crystalline dianthracene, $J$. Chem. Phys., 45, 3564-3567, 1966.

Cohen, M., L. Allamandola, A. G. G. M. Tielens, J. Bregman, J. P. Simpson, F. C. Witteborn, D. Wooden, and D. Rank, The infrared emission bands. I. Correlation studies and the dependence of $\mathrm{C} / \mathrm{O}$ ratio, Astrophys. J., 302, 737-749, 1986.

Colangeli, L., V. Mennella, G. A. Baratta, E. Bussoletti, and G. Strazzulla, Raman and infrared spectra of polycyclic aromatic hydrocarbon molecules of possible astrophysical interest, Astrophys. J., 396, 369377, 1992.

Dorren, J. D., M. Gudel, and E. F. Guinan, X-ray emission from the Sun in its youth and old age, Astrophys. J., 448, 431-436, 1995.

Duley, W. W. and D. A. Williams, The infrared spectrum of interstellar dust: Surface functional groups on carbon, Mon. Not. R. Astron. Soc., 196, 269-274, 1981.

Flickinger, G. C. and T. J. Wdowiak, Spectra of polycyclic aromatic hydrocarbon molecules at elevated temperature and the interstellar unidentified infrared bands, Astrophys. J., 362, L71-L74, 1990.

Flickinger, G. C., T. J. Wdowiak, and P. L. Gómez, On the state of the emitter of the 3.3 micron unidentified infrared band: Absorption spectroscopy of polycyclic aromatic hydrocarbon species, Astrophys. J., 380, L42-L46, 1991.

Frenklach, M., C. S. Carmer, and E. G. Feigelson, Silicon carbide and the origin of interstellar carbon grains, Nature, 339, 196-198, 1989.

Gillett, F. C., W. J. Forrest, and K. M. Merrill, 8-13-micron spectra of NGC 7027, BD+30 3639, and NGC 6572, Astrophys. J., 183, 87-93, 1973.

Hudgins, D. M. and L. J. Allamandola, Infrared spectroscopy of matrixisolated polycyclic aromatic hydrocarbons. 2 . The members of the thermodynamically most favorable series through coronene, J. Phys. Chem., 99, 3033-3046, 1995.

Hudgins, D. M. and S. A. Sandford, Infrared spectroscopy of matrix isolated polycyclic aromatic hydrocarbons. 1. PAHs containind two to four rings, J. Phys. Chem. A, 102, 329-343, 1998.

Hudgins, D. M., S. A. Sandford, and L. J. Allamandola, Infrared spectroscopy of polycyclic aromatic hydrocarbon cations. 1. Matrix-isolated naphthalene and perdeuterated naphthalene, J. Phys. Chem., 98, 42434253, 1994.

Jäger, C., S. Krasnokutski, A. Staicu, and F. Huisken, Identification and spectral properties of polycyclic aromatic hydrocarbons in carbonaceous soot produced by laser pyrolysis, Astrophys. J. Suppl. Ser., 166, 557-566, 2006.

Joblin, C., P. Boissel, A. Léger, L. d'Hendecourt, and D. Défourneau, Infrared spectroscopy of gas-phase PAH molecules, Astron. Astrophys., 299, 835-846, 1995.

Jura, M., The role of dust in ciecumstellar chemistry, in Astrochemistry; Proceedings of the International Astronomical Union Symposium, edited by M. S. Vardya and S. P. D. Tarafdar, 547-553 pp., D. Reidel Publishing Co., Dordrecht, 1987.

Kaito, C., Coalescence growth of smoke particles prepared by a gasevaporation technique, Jpn. J. Appl. Phys., 17, 601-609, 1978.

Kakudate, T., N. Yoshimoto, and Y. Saito, Polymorphism in pentacene thin film on $\mathrm{SiO}_{2}$ substrate, Appl. Phys. Lett., 90, 081903, 2007.

Kimoto, K. and I. Nishida, An electron diffraction study on the crystal structure of a new modification of chromium, J. Phys. Soc. Jpn., 22, 744-756, 1967.

Kimoto, K., Y. Kamiya, M. Nonoyama, and R. Uyeda, An electron microscope study on fine metal particles prepared by evaporation in argon gas at low pressure, Jpn. J. Appl. Phys., 2, 702-713, 1963.

Koga, K., T. Ikeshoji, and K. Sugawara, Size- and temperature-dependent structural transitions in gold nanoparticles, Phys. Rev. Lett., 92, 115507, 2004.

Léger, A. and J. L. Puget, Indentification of the 'unidentified' IR emission features of interstellar dust?, Astron. Astrophys., 137, L5-L8, 1984. 
Li, A., Interaction of nanoparticles with radiation, in Astrophysics of Dust, ASP Conf. Ser., 309, 417-452, 2004.

Mason, R., The crystallography of anthracene at $95^{\circ} \mathrm{K}$ and $290^{\circ} \mathrm{K}$, Acta Crystallogr., 17, 547-555, 1964.

Mathis, J. S., P. G. Mezger, and N. Panagia, Interstellar radiation field and dust temperatures in the diffuse interstellar matter and in giant molecular clouds, Astron. Astrophys., 128, 212-229, 1983.

Mennella, V., G. A. Baratta, A. Esposito, G. Ferini, and Y. J. Pendleton, The effects of ion irradiation on the evolution of the carrier of the 3.4 micron interstellar absorption band, Astrophys. J., 587, 727-738, 2003.

Mori, H., M. Komatsu, K. Takeda, and H. Fujita, Spontaneous alloying of copper into gold atom clusters, Philos. Mag. Lett., 63, 173-178, 1991.

Moutou, C., A. Léger, and L. d'Hendecourt, Far-infrared emission of PAH molecules (14-40 mm); a preparation for ISO spectroscopy, Astron. Astrophys., 310, 297-308, 1996.

Onaka, T., I. Yamaura, T. Tanabé, T. L. Roellig, and L. Yuen, Detection of the mid-infrared unidentified bands in the diffuse galactic emission by IRTS, Publ. Astron. Soc. Jpn., 48, L59-L63, 1996.

Oomens, J., J. M. Bakker, B. G. Sartakov, G. Meijer, and G. von Helden, the infrared spectrum of the benzoyl cation, Chem. Phys. Lett., 367, 576-580, 2003.

Peeters, E., S. Hony, C. Van Kerckhoven, A. G. G. M. Tielens, L. J. Allamandola, D. M. Hudgins, and C. W. Bauschlicher, The rich 6 to 9 $\mu \mathrm{m}$ spectrum of interstellar PAHs, Astron. Astrophys., 390, 1089-1113, 2002.

Prasad, S. S. and S. P. Tarafdar, UV radiation field inside dense clouds: Its possible existence and chemical implications, Astrophys. J., 267, 603609,1983

Radziszewski, J. G. and J. Michl, Symmetry assignment of vibrations in anthracene, phenazine, and acridine from infrared dichroism in stretched polyethylene, J. Chem. Phys., 82, 3527-3533, 1985.

Ramdas, S., G. M. Parkinson, and J. M. Thomas, Determination of crystal structure of metastable anthracene by a novel method, Nature, 284, 153$154,1980$.

Rapacioli, M., C. Joblin, and P. Boissel, Spectroscopy of polycyclic aromatic hydrocarbons and very small grains in photodissociation regions, Astron. Astrophys., 429, 193-204, 2005.

Rapacioli, M., F. Calvo, C. Joblin, P. Parneix, D. Toublanc, and F. Spiegelman, Formation and destruction of polycyclic aromatic hydrocarbon clusters in the interstellar medium, Astron. Astrophys., 460, 519-531, 2006.
Roche, P. F. and D. K. Aitken, 8-13 mm spectrophotometry of galaxiesV. The nuclei of five spiral galaxies, Mon. Not. R. Astron. Soc., 213, 789-797, 1985.

Rogers, C., M. H. Heyer, and P. E. Dewdney, HI, CO, and IRAS observations of NGC 7023, Astrophys. J., 442, 694-713, 1995.

Russell, R. W., B. T. Soifer, and K. M. Merrill, Observations of the unidentified 3.3 micrometer emission feature in nebulae, Astrophys. J., 213, 66-70, 1977a.

Russell, R. W., B. T. Soifer, and S. P. Willner, The 4 to 8 micron spectrum of NGC 7027, Astrophys. J., 217, L149-L153, 1977b.

Sakata, A., S. Wada, T. Tanabé, and T. Onaka, Infrared spectrum of the laboratory-synthesized quenched carbonaceous composite (QCC): Comparison with the infrared unidentified emission bands, Astrophys. J., 287, L51-L54, 1984.

Sakon, I., T. Onaka, T. Wada, Y. Ohyama, H. Matsuhara, H. Kaneda, Y. Ita, S. Ohyabu, H. Kataza, N. Fujishiro, C. Ihara, M. Ishigaki, D. Ishihara, W. Kim, Y. Okada, T. Takagi, T. Tanabé, K. Uemizu, M. Ueno, F. Usui, H. Watarai, B.-C. Koo, S. Serjeant, T. Nakagawa, T. Matsumoto, and H. Murakami, Properties of UIR bands in NGC 6946 based on mid-infrared imaging and spectroscopy with infrared camera on board AKARI, Publ. Astron. Soc. Jpn., 59, S483-S495, 2007.

Singh, S. and C. Sandoefy, Infrared spectra of photodimers of anthracene, benzo[a]anthracene, and tetracene, Can. J. Chem., 47, 257-263, 1969.

Szczepanski, J., D. Roser, W. Personette, M. Eyring, R. Pellow, and M. Vala, Infrared spectrum of matrix-isolated naphthalene radical cation, $J$. Phys. Chem., 96, 7876-7881, 1992.

Tielens, A. G. G. M., The Physics and Chemistry of the Interstellar Medium, pp. 215, Cambridge University Press, Cambridge, 2005.

Tielens, A. G. G. M., S. Hony, C. van Kerckhoven, and E. Peeters, Interstellar and circumstellar PAHs, in The Universe as Seen by ISO, edited by P. Cox and M. F. Kessler, 579 pp., ESA SP-427, 1999.

van Diedenhoven, B., E. Peeters, C. Van kerckhoven, S. Hony, D. M. Hudgins, L. J. Allamandola, and A. G. G. M. Tielens, The profiles of the 3-12 micron polycyclic aromatic hydrocarbon features, Astrophys. J., 611, 928-939, 2004.

Willner, S. P., B. T. Soifer, and R. W. Russell, 2 to 8 micron spectrophotometry of M82, Astrophys. J., 217, L121-L124, 1977.

M. Saito, I. Sakon, C. Kaito, and Y. Kimura (e-mail: ykimura@m.tains.tohoku.ac.jp) 